\title{
Defect Analysis and Alignment Quantification of Line Arrays Prepared by Directed Self-assembly of a Block Copolymer
}

\author{
C. Simão, ${ }^{* a}$ D. Tuchapsky, ${ }^{\mathrm{b}}$ W. Khunsin, ${ }^{\mathrm{a}, \mathrm{c}}$ A. Amann, ${ }^{\mathrm{b}}$ M. A. Morris ${ }^{\mathrm{d}, \mathrm{e}}$ and C. M. Sotomayor Torres ${ }^{\mathrm{a}, \mathrm{f}}$
}

${ }^{a}$ Catalan Institute of Nanoscience and Nanotechnology ICN2, Campus de la UAB, Barcelona 08193, Spain; ${ }^{\mathrm{b}}$ School of Mathematics and the Tyndall National Institute, UCC, Cork Ireland; ${ }^{\mathrm{c}}$ Green Nanotechnology Research Centre, Research Institute for Electronic Science, Hokkaido University, 0010021 Sapporo, Japan; ${ }^{\mathrm{d}}$ School of Chemistry and the Tyndall National Institute, ${ }^{\mathrm{e}}$ UCC, Cork Ireland; Centre for Research on Adaptive Nanostructures and Nanodevices, TCD, Ireland; ${ }^{\mathrm{f}}$ Catalan Institute of Research and Advanced Studies, Barcelona 08010 , Spain;

\begin{abstract}
Different linear patterns obtained from the directed self-assembly of the block copolymer (BCP) polystyrene-b-polyethylene oxide (PS-b-PEO) were analysed and compared. The hexagonal phase PS-b-PEO in a thin film exhibits linear pattern morphology, by conventional solvent annealing in an atmosphere saturated in chloroform. The surface energy of the silicon substrates was varied using surface functionalization of a self-assembly monolayer (SAM) and a polymer brush, chosen to investigate the influence of the surface energy on the self-assembly of the BCP. The linear patterns formed were analyzed with innovative image analysis software specifically developed in our laboratory to identify elements and defects of line arrays from block copolymer self-assembly. The technique starts by performing dimensional metrology to calculate the pitch size and estimate the linewidth of the lines. Secondly, the methodology allows identification and quantification of typical defects observable in BCP systems, such as turning points, disclination or branching points, break or lone points and end points. The defect density and the quantification of the alignment were estimated using our technique. The methodology presented here represents a step forward in dimensional metrology and defect analysis of BCP DSA systems and can be readily used to analyze other lithographic or non-lithographic patterns.
\end{abstract}

\section{Introduction}

At the sub-20 nm scale nanostructures, fabrication costs have triggered the search of alternative fabrication techniques. One of the most promising technologies is directed self-assembly of block copolymers,[1] which are valuable systems to create hexagonal and linear patterns on surface, only by tuning substrate surfaces properties.[2-4]

Extending the limits of nanofabrication to the nanometre scale had impact on specialized metrology suitable both to the dimensions and novel materials requirements.[5-7] This self-assembly type technology can result in characteristic fingerprint patterns to highly controllably aligned lines patterns on surface using state-of-the-art directed self-assembly (DSA) techniques.[8-11] These procedures rely on the control of surface properties, either chemical or topological, to create a confinement commensurable with the polymer chains and have a direct impact in their regular arrangement.

One of the biggest challenges towards industrial adaptation that remains to be addressed is the estimation of defect density on these systems. Here we present an innovative methodology to analyse linear patterns generated by BCP DSA,[12] based on an image analysis software that identifies the line elements and is able to provide valuable identification of typical defects

Metrology, Inspection, and Process Control for Microlithography XXVIII, edited by Jason P. Cain, Martha I. Sanchez, Proc. of SPIE Vol. 9050, 905028 · @ 2014 SPIE · CCC code: 0277-786X/14/\$18 · doi: 10.1117/12.2046075 
from BCP DSA, namely turns, dislocations, lone and branching points, all of which are crucial for the optimization and tailoring of the assembly process to obtained desired structures.. The defect density and the quantification of the alignment are then estimated based on the statistical data gathered by the image analysis.

\section{Experimental}

\subsection{Materials}

Block copolymer polystyrene-b-polyethylene oxide (PS-b-PEO) $\left(42 \mathrm{~kg} \cdot \mathrm{mol}^{-1}\right.$ - $11.5 \mathrm{~kg} \cdot \mathrm{mol}^{-1}$ ) and polystyrene hydroxy terminated (PS-OH) (Mw 5000 g.mol ${ }^{-1}$ ) were used as purchased from Polymer Source Inc. (USA). Trimethoxy silane (TMS) $(95 \%)$ and solvents were used as purchased from Sigma-Aldrich (Madrid, Spain). $100 \mathrm{~mm}$ silicon wafers (p type, boron, $<100>, 10-20 \mathrm{ohm} / \mathrm{cm}$ ) (Silicon Materials Inc. USA), were used as purchased.

\subsection{Silicon substrates functionalization and characterization}

Silicon substrates for functionalization were first degreased by rinsing with a series of solvents (acetone, ethanol and isopropanol) and then treated in a piranha bath $\left(\mathrm{H}_{2} \mathrm{SO} 4: \mathrm{H}_{2} \mathrm{O}_{2}\right)(3: 1)$ for 1 minute to achieve a homogenous silanol layer on the surface.[13] For self-assembly monolayer (SAM) functionalization, the piranha treated silicon wafer was exposed to TMS vapours for 10 minutes. After this time, the wafer was washed thoroughly with toluene, followed by ethanol and dried under a nitrogen stream. For polymer brush treatment, the piranha treated silicon wafer was spincoated with a $1 \%$ (w/w) PS$\mathrm{OH}$ solution in toluene at $3000 \mathrm{rpm}$ for 30 seconds and placed in an oven at $180^{\circ} \mathrm{C}$ for 5 hours. After cool down to room temperature, the polymer brush treated wafer was washed thoroughly with toluene, ethanol and dried under nitrogen stream.

\subsection{Block copolymer sample preparation}

A solution of PS-b-PEO $1 \%(\mathrm{w} / \mathrm{w})$ in toluene was prepared and filtered through a $200 \mathrm{~nm}$ pore size syringe filter. This solution was dispensed over three silicon substrates (bare Si, TMS SAM Si and polymer brush Si) and spincoated at 3000 rpm for 30 seconds. The samples were immediately placed inside a closed vessel with an atmosphere saturated in chloroform vapours for three hours to undergo solvent annealing. After this time, the samples were removed from the vessel and dried with a gentle nitrogen stream.

\subsection{Characterization}

A goniometer (SUSS Microtech) was used to record and measure the contact angle of the different surfaces used and prepared. The samples were imaged as prepared by field-emission scanning electron microscopy (SEM) in a Zeiss Merlin microscope with a Gemini II column, with operating electron energy of $1.2 \mathrm{keV}$. The block copolymer patterns were analyzed by the directed self-assembly image analysis software developed in the laboratory applied to the collected SEM images. The code is written in Python ${ }^{(\mathrm{TM})}$ language for version 2.7.5, and was run in a conventional desktop terminal with Windows XP (Intel $^{\circledR}$ Core $^{(\mathrm{TM})} 2 \mathrm{CPU} @ 1.86 \mathrm{GHz}$ and $3072 \mathrm{MB}$ RAM). Using this computational power, the time of analysis takes about 5 minutes for SEM image (1260x1260 pixel resolution). 


\section{Results and Discussion}

The silicon substrates were characterized by contact angle and the values obtained summarized in Table 1 . The different hydrophobicity of the three differently functionalised surfaces can be readily seen.

Table 1. Summary of silicon substrates contact angle values.

\begin{tabular}{|lc|}
\hline \multicolumn{2}{|c|}{ Water contact angles } \\
Water contact angles \\
Bare Si & $45 \mathrm{deg}$ \\
TMS SAM/Si & $65 \mathrm{deg}$ \\
PS brush/Si & $83 \mathrm{deg}$ \\
\hline
\end{tabular}

The block copolymer solutions were spincoated over the three different substrates and immediately solvent annealed (Scheme 1). As observable in Figure 1, this block copolymer shows spontaneous microphase segregation upon spincoating, resulting in cylinders perpendicular to substrate embedded in the PS matrix, resulting in the observable hexagonal dot array. PS-b-PEO systems are well-known for their good microphase segregation in brushless substrates.[14] After annealing in a saturated atmosphere of chloroform, the PEO cylinders flip the orientation towards parallel to substrate, resulting in a line pattern. The cyclical flipping behaviour has been reported in this BCP system, but with toluene as annealing solvent.[15]

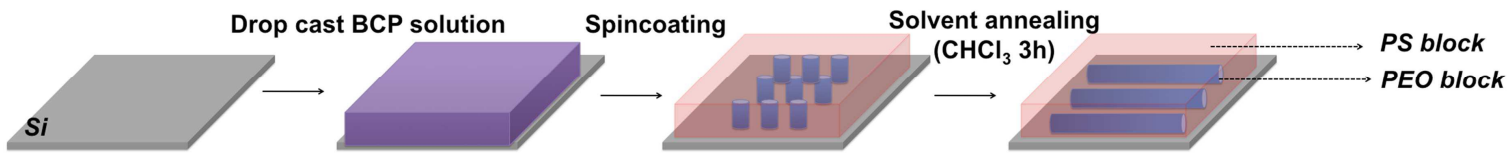

Scheme 1. Schematic workflow of the preparation of line patterns from PS-b-PEO DSA.

From the SEM images measurements, the critical dimensions of this BCP system have a periodicity of around $40 \mathrm{~nm}$ and diameter of the cylinders of around $20 \mathrm{~nm}$. 


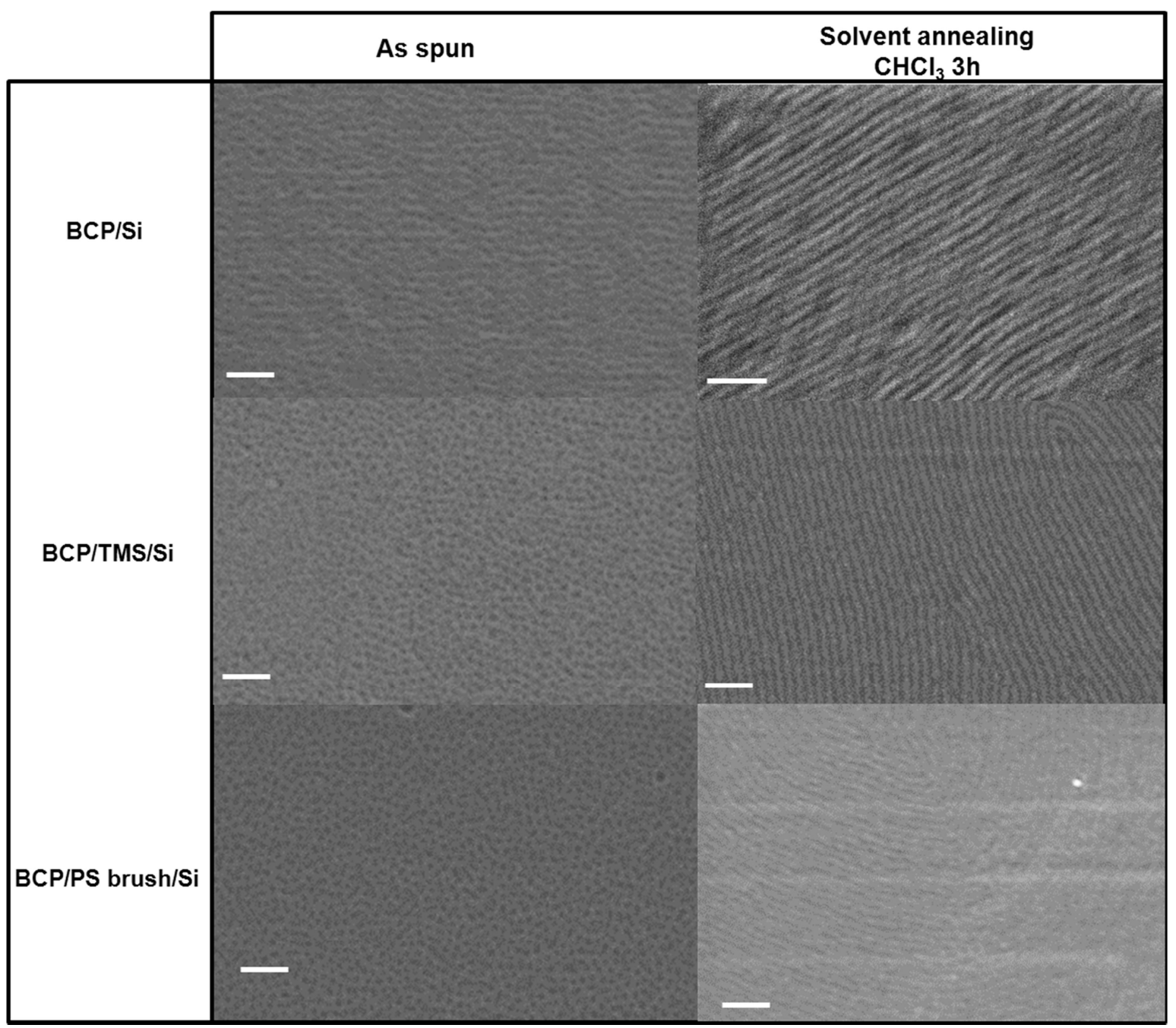

Figure 1. SEM images of the block copolymer samples as spun and after solvent annealing. The scale bar represents $200 \mathrm{~nm}$.

In general, the alignment yield ranges several BCP periods in the three substrates, confirming that chloroform annealing is a suitable DSA procedure to produce linear patterns with the present PS-PEO system. Nevertheless, several defects are spotted randomly in all samples and it is difficult to conclude if the surface free energy or functionalization (in TMS and brush cases) of the substrates have an impact in the DSA of the block copolymer.

To overcome this problem, the line patterns recorded by FE-SEM were evaluated with our directed self-assembly image analysis software. The code has been developed to identify lines and defects specific from block copolymer self-assembly. In the first step, the region of interest is chosen in the SEM image. The first computational step identifies the elements (lines) and four type of DSA defects (lone or break points, branching, dislocation and turning points) and are colour-coded as shown in (Figure 2). 


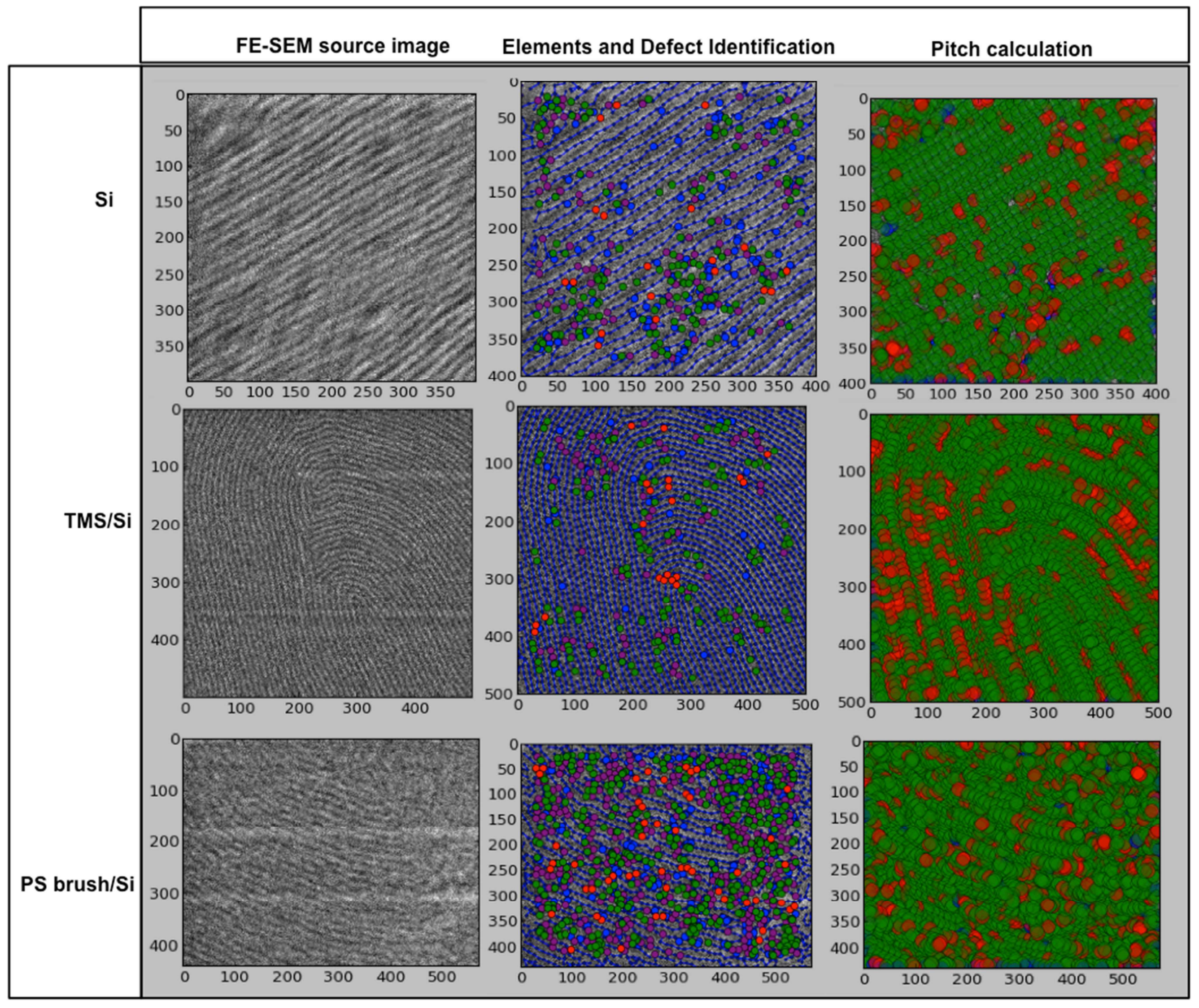

Pixel x Pixel

Pixel x Pixel

Pixel x Pixel

Figure 2. Left panel: original SEM images of the block copolymer samples after solvent annealing. Middle panel: software output of element (dark blue dashed dots) and defect identification (colour-coding: end points (green), lone points (blue), branch points (purple) and turn points (red)). Right panel: software output of pitch estimation graphic representation: the green and red dots represent the points used and excluded from the calculation, respectively.

The next stage in our analysis consists in extracting the pitch size distribution (Figure 3), and the estimated values and deviations collected are summarized in Table 2 . The present methodology has been specially developed to calculate the pitch size, while the linewidths are indicative estimations. This can be observed in the associated error to these two variables; 
while the pitch size estimations have deviations below $10 \%$ in images of $\mathrm{BCPs}$, the linewidths have standard deviations slightly above $10 \%$.
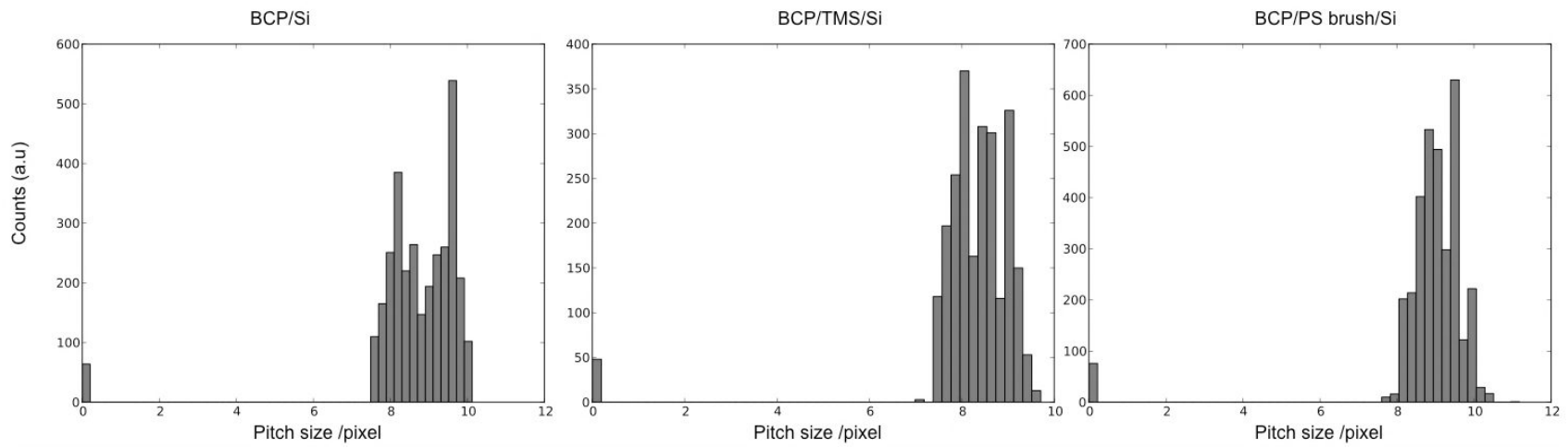

Figure 3. Pitch size distribution histograms calculated from the image analysis code for the three samples.

Table 2. Dimensional analysis of the line patterns by the image analysis methodology.

\begin{tabular}{|cccc|}
\hline \multicolumn{4}{c|}{ DSA Line Pattern Dimensional Analysis } \\
Substrate & Analysed area $\left(\mathrm{cm}^{2}\right)$ & Pitch average $/ \mathrm{nm}$ & Linewidth estimation/nm \\
\hline $\mathrm{Si}$ & $2.53 \times 10^{-9}$ & $43.5 \pm 4.2$ & $20.1 \pm 3.2$ \\
$\mathrm{TMS} / \mathrm{Si}$ & $4.10 \times 10^{-9}$ & $42.1 \pm 3.2$ & $19.5 \pm 3.7$ \\
$\mathrm{PS} / \mathrm{Si}$ & $4.02 \times 10^{-9}$ & $42.4 \pm 3.4$ & $21.2 \pm 5.6$ \\
\hline
\end{tabular}

Once the initial analysis of dimensional metrology based on the SEM image has been completed, the following step deals with statistical analysis of the extracted data.

The defects are identified as coloured dots and overlaid with the original SEM image and the marked lines, as seen in Figure 2. The collected statistics concerning the identification and quantification of the defects is summarized in Figure 4. 


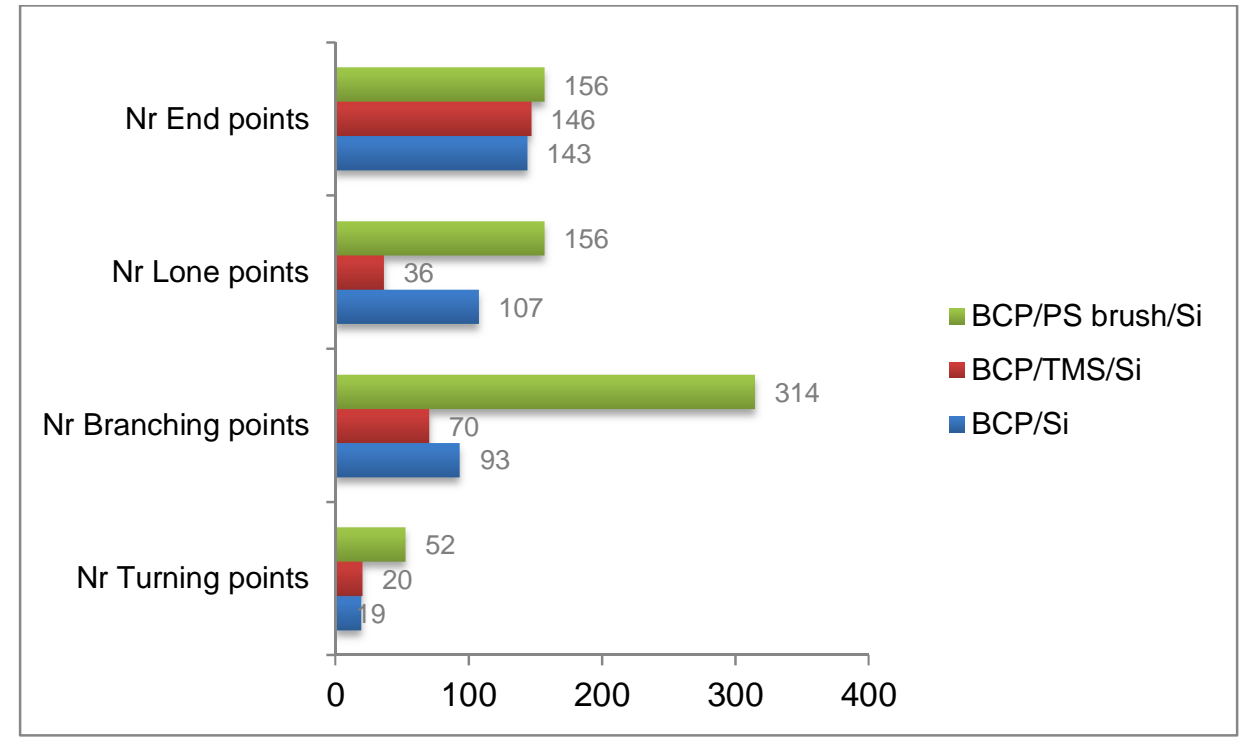

Figure 4. Type and number of defects found in the three samples.

With basis on the quantification of the four fundamental defects, the defect density can be defined as the total number of defects found in the analysed area, and was calculated for the three samples following Equation 1.

$$
\text { DefectDensity }=\frac{N r \cdot \text { Branching }+N r . T u r n i n g+N r . \text { Lone }+N r E n d}{\text { Area }} \quad \text { Eq. } 1
$$

Furthermore, the information gathered in the present methodology enables us to estimate the order of the linear pattern. If we consider the perfect order an array of fully straight lines, we can describe it as a100\% aligned line array. Thus, the alignment percentage of a linear array can be described as the density of turning points in relation to the total number of lines found, as defined in Equation 2.

$$
\% \text { Alignment }=\left(1-\frac{\text { Nr.Turns }}{\text { NrLines }}\right) \times 100 \quad \text { Eq. } 2
$$

The data concerning the defect analysis of the linear arrays investigated here are summarised in Table 3. 
Table 3. Summary of number of elements, defects and estimated defect density in each sample.

\begin{tabular}{|cccccc|}
\hline \multicolumn{5}{c|}{ DSA Line Pattern Defect Analysis } \\
Substrate & $\begin{array}{c}\text { Analysed } \\
\text { area }\left(\mathrm{cm}^{2}\right)\end{array}$ & $\begin{array}{c}\text { Total } \mathrm{nr} \\
\text { lines }\end{array}$ & $\begin{array}{c}\text { Total } \mathrm{nr} \\
\text { defects }\end{array}$ & $\begin{array}{c}\text { Defect Density } \\
\left(\text { nr defects } \times 10^{9} / \mathrm{cm}^{2}\right)\end{array}$ & Alignment \% \\
\hline $\mathrm{Si}$ & $2.53 \times 10^{-9}$ & 330 & 362 & 143 & 94.2 \\
$\mathrm{TMS} / \mathrm{Si}$ & $4.10 \times 10^{-9}$ & 170 & 272 & 66.4 & 88.2 \\
$\mathrm{PS} / \mathrm{Si}$ & $4.02 \times 10^{-9}$ & 370 & 678 & 168 & 85.9 \\
\hline
\end{tabular}

In Figure 5, the defect density and the alignment percentage are graphically represented for a convenient comparison, with units as described in Table 2. It is observable that the lowest defect density and best aligned array corresponds to the PS-bPEO sample grown on the TMS SAM functionalized silicon substrate.

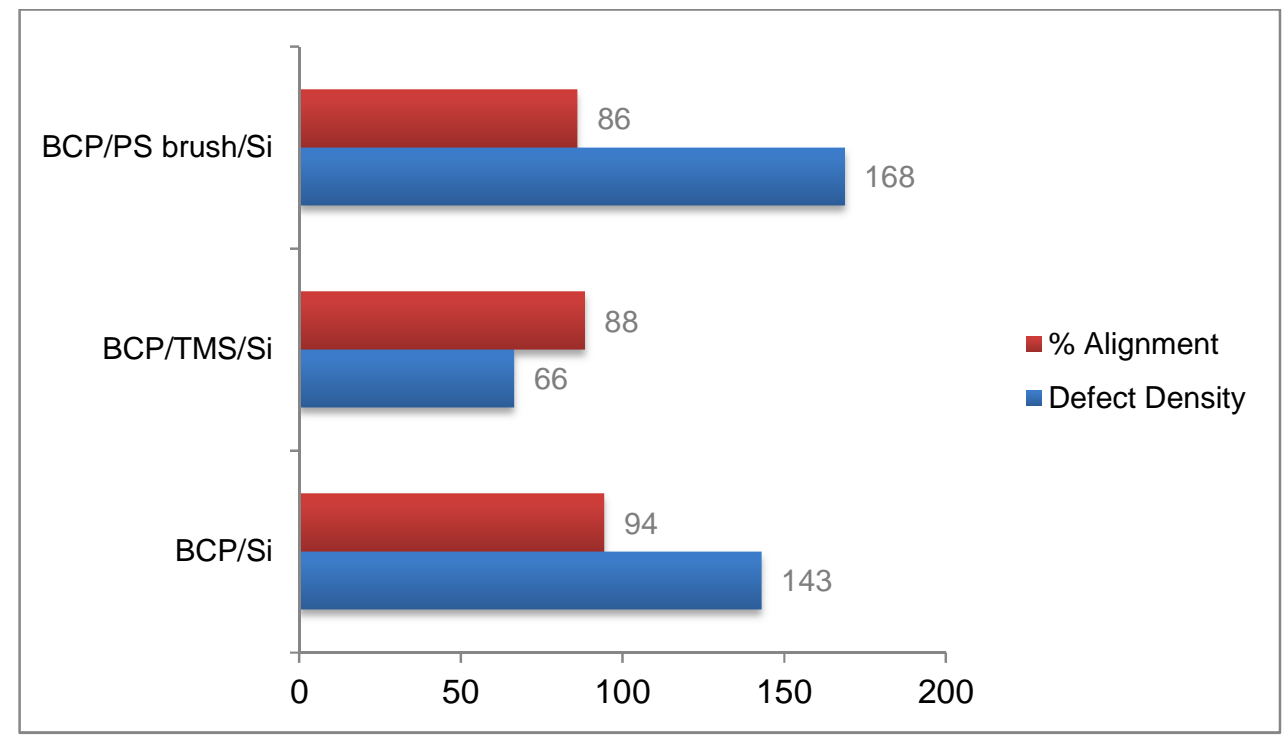

Figure 5. Comparison between defect density (nr defects $\times 10^{9} / \mathrm{cm}^{2}$ ) and alignment yield in percentage of the three samples studied. 


\section{Conclusions}

The directed self-assembly of the hexagonal phase of PS-b-PEO treated with chloroform vapour results in PEO cylinders orientated parallel to substrate in a PS matrix. That orientation produces rather homogeneous line arrays in a cost-effective procedure. The line array examination was performed with the computational image analysis method that identifies the lines in the array along four type of defects. The dimensional analysis of the patterns yields an approximation of the pitch size of the PS-b-PEO system used, giving an average $42 \mathrm{~nm}$ pitch size value with a standard deviation below $10 \%$. This calculation is rather good, given the simplicity of the method, the low computational power needed and the quality of the SEM images.

Furthermore, the defect analysis obtained from the method permitted estimating the defect density, making it possible to statistically quantify the order of the line patterns within the experimental error, the percentage of alignment, based on the density of turn points in the line array. The defect density and the percentage of alignment of the investigated samples demonstrate that the best rated sample is the PS-b-PEO on a TMS SAM functionalized silicon substrate due to its lowest defect density with $66.4 \times 10^{9}$ defects per $\mathrm{cm}^{2}$ with $88.2 \%$ alignment yield.

It has been demonstrated here that this technique is simple, versatile and powerful metrological tool for quality control of line arrays prepared by BCP DSA. Its fair accuracy combined with its simplicity and features dedicated to BCP DSA makes is it a valuable tool to standardize BCP DSA systems, which may be key for the successful implementation of the BCP lithography technology in the established semiconductor industry.

\section{Acknowledgments}

The research leading to these results received funding from the European Union FP7 under the project LAMAND (grant agreement $n^{\circ}$ 245565), the Spanish MICIN under the project TAPHOR (contract nr. MAT2012-31392) and the Science Foundation Ireland under grant number 09/SIRG/I1615. The authors also wish to thank the Microscopy Division of ICN2 for the technical support on FE-SEM. The contents of this work are the sole responsibility of the authors.

\section{References}

[1] C. Park, J. Yoon, and E. L. Thomas, "Enabling nanotechnology with self assembled block copolymer patterns," Polymer, 44(22), 6725-6760 (2003).

[2] Y. S. Jung, J. B. Chang, E. Verploegen et al., "A path to ultranarrow patterns using self-assembled lithography," Nano Letters, 10(3), 1000-1005 (2010).

[3] K. W. Gotrik, A. F. Hannon, J. G. Son et al., "Morphology control in block copolymer films using mixed solvent vapors," ACS Nano, 6(9), 8052-8059 (2012).

[4] T. Ghoshal, R. Senthamaraikannan, M. T. Shaw et al., "In situ hard mask materials: A new methodology for creation of vertical silicon nanopillar and nanowire arrays," Nanoscale, 4(24), 7743-7750 (2012).

[5] R. Gronheid, L. Van Look, P. Rincon Delgadillo et al., "High throughput grating qualification for rating directed self-assembly pattern performance using optical metrology," Journal of Photopolymer Science and Technology, 26(2), 147-152 (2013).

[6] M. Isawa, K. Sakai, P. A. Rincon Delgadillo et al., "Line edge roughness measurement technique for fingerprint pattern in block copolymer thin film." 8681, 868114

[7] P. Rincon Delgadillo, R. Harukawa, M. Suri et al., "Defect source analysis of directed self-assembly process (DSA of DSA)." 8680, 86800L 
[8] M. Salaun, M. Zelsmann, S. Archambault et al., "Fabrication of highly ordered sub-20 nm silicon nanopillars by block copolymer lithography combined with resist design," J. Mater. Chem. C, 1(22), 3544-3550.

[9] D. Borah, C. D. Simao, R. Senthamaraikannan et al., "Soft-graphoepitaxy using nanoimprinted polyhedral oligomeric silsesquioxane substrates for the directed self-assembly of PS-b-PDMS," European Polymer Journal.

[10] S. Ouk Kim, H. H. Solak, M. P. Stoykovich et al., "Epitaxial self-assembly of block copolymers on lithographically defined nanopatterned substrates," Nature, 424(6947), 411-414 (2003).

[11] C. Simão, A. Francone, D. Borah et al., "Soft graphoepitaxy of hexagonal PS-b-PDMS on nanopatterned POSS surfaces fabricated by nanoimprint lithography," Journal of Photopolymer Science and Technology, 25(2), 239-244 (2012).

[12] C. M. Sotomayor-Torres, C. Simao, A. Amann et al., [European Patent Application ], (2013).

[13] C. Simao, M. Mas-Torrent, V. Andre et al., "Intramolecular electron transfer in the photodimerisation product of a tetrathiafulvalene derivative in solution and on a surface," Chemical Science, 4(1), 307-310 (2013).

[14] P. Mokarian-Tabari, T. W. Collins, J. D. Holmes et al., "Brushless and controlled microphase separation of lamellar polystyrene-b-polyethylene oxide thin films for block copolymer nanolithography," Journal of Polymer Science, Part B: Polymer Physics, 50(13), 904-909 (2012).

[15] P. Mokarian-Tabari, T. W. Collins, J. D. Holmes et al., "Cyclical "flipping" of morphology in block copolymer thin films," ACS Nano, 5(6), 4617-4623 (2011).

*claudia.simao@icn.cat; phone +34937371616; www.icn.cat/ p2n 